\title{
Surface Morphology Analysis of Composite Thin Films based on Titanium-Dioxide Nanoparticles
}

\author{
Ş. Ţălu ${ }^{a}$, M. Bramowicz ${ }^{b}$, S. Kulesza ${ }^{c}$, F. Pignatelli ${ }^{d}$ And M. Salerno ${ }^{e, *}$ \\ ${ }^{a}$ Technical University of Cluj-Napoca, Faculty of Mechanical Engineering, Department of AET, Discipline of \\ Descriptive Geometry and Engineering Graphics, 103-105 B-dul Muncii St., Cluj-Napoca 400641, Cluj, Romania \\ ${ }^{b}$ University of Warmia and Mazury in Olsztyn, Faculty of Technical Sciences, \\ M. Oczapowskiego 11, 10-719 Olsztyn, Poland \\ ${ }^{c}$ University of Warmia and Mazury in Olsztyn, Faculty of Mathematics and Computer Science, \\ Sloneczna 54, 10-710 Olsztyn, Poland \\ ${ }^{d}$ Center for Micro-BioRobotics, Istituto Italiano di Tecnologia, \\ viale Rinaldo Piaggio 34, I-56025 Pontedera (Pisa), Italy \\ ${ }^{e}$ Istituto Italiano di Tecnologia, Materials Characterization Facility, \\ via Morego 30, I-16163 Genova, Italy \\ (Received December 14, 2016; in final form March 14, 2017)

\begin{abstract}
This study addressed the advanced micro-morphological characterization of 3D surfaces of thin films based on composites of an organic matrix of poly-methyl-methacrylate and inorganic titanium-dioxide nanocrystals. The films were prepared by spin-coating of a series of solutions with increasing loading concentration of the nanocrystals, from 5 up to $40 \%$ by weight. The film surfaces were investigated by atomic force microscopy, and the resulting images were analyzed in detail to obtain a full quantitative description of their surface micromorphology. The fractal analysis of the surface roughness was used to extract the characteristics of amplitude, spatial distribution, and pattern. A fractal geometry was indeed observed, and the corresponding fractal dimension $D$ was determined for all the samples. In view of the possible use of these thin films as functional coatings, the calculated quantities are discussed with respect to the foreseen durability of these surfaces during operation under wear.
\end{abstract}

DOI: 10.12693/APhysPolA.131.1529

PACS/topics: 68.37.Ps, 64.60.al, 68.35.Ct, 68.55.jm, 62.23.Pq

\section{Introduction}

The field of composites has presented the most important evolution of the plastic technology and the materials science in general during the recent decades [1]. Composite materials are obtained by mixing an inorganic particulate phase (fillers) into an organic paste (monomer to be polymerized), along with additives, mostly also of organic nature, playing different accessory roles (bonding agents, pigments, possible photo-initiators etc. [2]). This process gives rise to a novel material which should present a combination of useful properties from both chemical-physical phases [1,3]. Especially for the filler particles the possible choices are widespread, since both the particle composition and size can be varied, and even provided in multiple populations such as it happens for e.g. the dental restorative composites [4, 5].

As soon as the nanotechnology has made new nanoparticles available, one of the first options of the materials scientists has always been to mix them into well-known matrixes, and check for the resulting properties. This was formerly the case, for example, for carbon nanotubes [6], and recently the same is occurring also for

*corresponding author; e-mail: marco.salerno@iit.it graphene flakes $[7,8]$. Here we report on the morphological properties of composites obtained by dispersing in a common polymer, poly-methyl-methacrylate (PMMA), elongated nanocrystals of titanium-dioxide (titania). Nanostructured titania is of interest for several applications [9], among which are photocatalytic degradation of pollutants [10] and biomedical implant surfaces [11].

Obviously, when mixing a particle filler into the polymer to make a composite, proper engineering is required in terms of optimum dispersion of the fillers, to avoid aggregation which would not provide uniform composite properties and can even degrade the resin matrix performance instead of augmenting it. This is even more the case for thin films of composites, used in coating applications, such as the samples investigated in this work $[12,13]$. To fully characterize the surface micromorphology of our samples, we have measured them by means of atomic force microscopy (AFM), which allows for a direct $3 \mathrm{D}$ imaging in the real space, and have carried out a thorough analysis of the resulting images, taking advantage of fractal theory. Fractal geometry is a well-known tool for quantification of irregular complex structures and patterns across many spatial or temporal scales, relevant to applications in surface engineering research [14-16]. In particular, the combination of both above-mentioned techniques, namely AFM for imaging and fractal geometry for analysis, has already 
demonstrated to be a powerful tool in the investigation of the surface morphology of different materials (e.g. silicon carbides [17] and aluminum nitrides [18]), and is expected to give deeper insight in the complexity of the topographical patterns resulting from our nanocomposite thin films.

\section{Materials and methods}

\subsection{Nanocomposite films preparation}

The filler nanoparticles were nanocrystals of titania, which were prepared in our laboratory according to chemical synthesis previously described [19]. The primary nanocrystals had typical size of $\approx 30 \mathrm{~nm}$ length and $\approx 4 \mathrm{~nm}$ diameter. They were also coated with oleic acid surfactant, to avoid or limit aggregation, for an estimated content of $\approx 10 \mathrm{wt} \%$ to the inorganic phase. The nanoparticles suspended in toluene were mixed with poly-methyl-methacrylate (PMMA, Aldrich, Italy) with nominal $M_{w}=120 \mathrm{kDa}$. Solutions with different loading by weight of fillers to polymer were prepared, namely 0 (pure polymer), 5, 10, 20,30, and 40\%. The liquid composite was spin-coated on optical quality glass substrates, at $2000 \mathrm{rpm}$ for $1 \mathrm{~min}$ (open cover) and allowed to dry in ambient atmosphere. The film thickness, as measured with AFM on cross-sections of scratches made on purpose, was always in the order of $\approx 100 \mathrm{~nm}$.

\subsection{AFM imaging of the film surface}

The AFM scan size was set at $10 \mu \mathrm{m}$, with square scan area. All AFM measurements were carried out at room temperature $\left(25 \pm 1^{\circ} \mathrm{C}\right)$ and relative humidity $\leq 48 \%$, in tapping mode. As the image sampling was of $512 \mathrm{pixel} /$ line, the measurement resolution was $\approx 20 \mathrm{~nm} /$ pixel. The acquisitions were repeated three times in different areas, for each of two specimens prepared under the same conditions (i.e. representing the same sample). The resulting images were all checked visually for consistency, by the expert AFM user. Those with visible defects not assignable to the pristine or ideal sample surface (contaminants, air bubbles in the coated solution, dust) were discarded. Only one image per sample was selected to be representative, and sent to the analysis.

\subsection{Statistical analysis}

Statistical analysis on the AFM images was performed using the SPSS 14 for Windows (Chicago, Illinois, USA). One-way analysis of variance (ANOVA) was used to test the differences between the two groups with the Scheffé post-hoc tests for multiple comparisons. Differences with a $P$ value of 0.05 or less were considered statistically significant. The average $D$ results were expressed as mean value and standard deviation.

\subsection{Characterization of the surface texture}

AFM images obtained on any type of solid material can be utilized for the study of their surface texture by numerical derivation of statistical and fractal characteristics as the post-processing protocol. These methods demonstrated their applicability for studies of various structures, including: thin diamond films [20], Fe nanoparticles on foreign substrates [21], and even magnetic domains [22]. The statistical and fractal characteristics can be derived from the autocorrelation function $R$ (see Fig. 1A) defined as follows:

$$
\begin{aligned}
& R(m, n)=\frac{1}{(N-n)(N-m)} \\
& \quad \times \sum_{k=1}^{N-n} \sum_{l=1}^{N-m}(z(k+m, y+l) z(k, l)),
\end{aligned}
$$

where $N$ is the number of scan steps along given scan axis, $m$ and $n$ are integers describing the lag between given image and its copy.
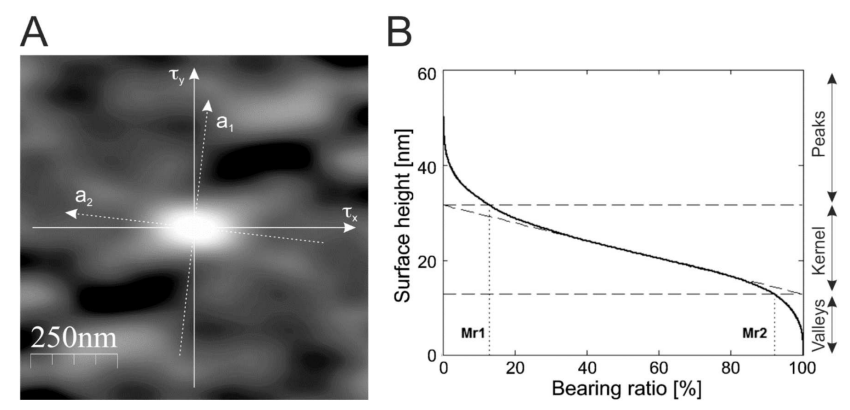

Fig. 1. (A) Example plot of the autocorrelation function $R$ with main directions of the anisotropy: $a_{1}$ and $a_{2}$. (B) Example Firestone-Abbott bearing curve for determining functional properties of the surface under study.

Even though real surfaces are formed in random processes, they might be anisotropic to a certain degree. Surface lay of this type can be characterized using the surface anisotropy ratio $S_{t r}$, defined as the fraction of extreme decay lengths $\tau$ along which autocorrelation function falls down from 1.0 to 0.2 [23]:

$$
0<S_{t r}=\frac{\tau_{a 1}}{\tau_{a 2}}
$$

where $a 1$, and $a 2$ are the axes of the fastest and the slowest autocorrelation decay, respectively (see Fig. 1A). As a rule of thumb, $S_{t r}$ higher than 0.5 corresponds to isotropic surface, while lower than 0.3 corresponds to highly anisotropic surface.

The surfaces under study can also be characterized with respect to their scaling behavior. To this end the autocorrelation function $R$ needs to be re-computed into structure function $S$, according to the formula [24]:

$$
S(m, n)=2 S_{q}^{2}(1-R(m, n)),
$$

where $S_{q}$ is the root-mean-square (RMS) surface roughness. Scaling laws define whether and how geometrical objects exhibit self-similar properties similar to fractals.

Thomas and Thomas [25] showed that the structure function averaged along an arbitrary direction could be fit with a power-law dependence in a form

$$
S(\tau)=K \tau^{2(2-D)},
$$


where $D$ is the fractal dimension, and $K$ is called pseudotopothesy. As a rule, $D$ influences the relative amplitude of surface variations over the wavelengths, whereas $K$ influences their absolute amplitude. However, with increasing scale length this power-law behavior steadily vanishes, and the structure function asymptotically reaches $2 S_{q}^{2}$ value. A point at which this dependence breaks down is referred to as the corner frequency $\tau_{c}$.

Functional parameters can be derived from the Firestone-Abbott bearing curve (see Fig. 1B). The curve appears after height samples are arranged in a descending order and plotted on the percent scale, where $100 \%$ corresponds to the lowest sample in the series.

DIN 4776 standard specifies several characteristics that can be useful in describing topographical complexity of the surface, including:

a) kernel roughness depth $S_{k}$ - thickness of the core at the flattest part of the bearing curve where the largest increase in material exists;

b) reduced peak height $S_{p k}$, reduced valley depth $S_{v k}$ - thickness of the bearing curve above/below the core profile, respectively;

c) upper bearing area $M_{r 1}$, lower bearing area $M_{r 2}$ intersection points of horizontal lines plotted from both ends of the flattest tangent of the bearing curve with that curve that delimit peaks and valleys from the core, respectively.

\section{Results and discussion}

Figure 2 shows $10 \mu \mathrm{m} \times 10 \mu \mathrm{m}$ area topography of the samples under study seen in AFM measurements. It can be seen in Fig. 2A that the surface topography of the pure PMMA film (0 wt\% of nanoparticle filler) only showed tiny spots, not higher than $\approx 10 \mathrm{~nm}$, placed on otherwise flat substrate. The diameter of the largest spots does not exceed $\approx 250 \mathrm{~nm}$. In turn, the sample with $5 \mathrm{wt} \%$ of fillers (see Fig. 2B) shows a large number of well-developed circular grains, all similar in size and shape (around $330 \mathrm{~nm}$ in diameter and $50 \mathrm{~nm}$ in height). Obviously, even if the filler nanoparticles are elongated (with aspect ratio as high as $\approx 7$ ), the corresponding features emerging on the surface of the film that embeds them are just symmetric tips. These probably correspond to the top-most end of particles tilted along the long axis with respect to the horizontal plane. Average grain density reaches $\approx 1.23 \times 10^{8} \mathrm{~cm}^{-2}$, and the particles show the tendency to partly align their tops in linear patterns made of 3-4 of them. These structures increase their tendency to assemble with increasing concentration, and for $10 \mathrm{wt} \%$ (see Fig. 2C), once the particles overlap, they form elongated blocks of irregular shapes, 2-3 $\mu \mathrm{m}$ long, and around $1 \mathrm{\mu m}$ wide. As this process proceeds further, the blocks become bigger (see Fig. 2D, sample with $20 \mathrm{wt} \%$ ), and join to form even bigger hill-like structures of more regular, although diffused shapes (see Fig. 2E, sample with $30 \mathrm{wt} \%$ ). As seen in Fig. 2F (sample with $40 \mathrm{wt} \%$ particles), the growth process ends up leaving a wavy surface, composed of rounded hills larger than $5 \mu \mathrm{m}$ in diameter.
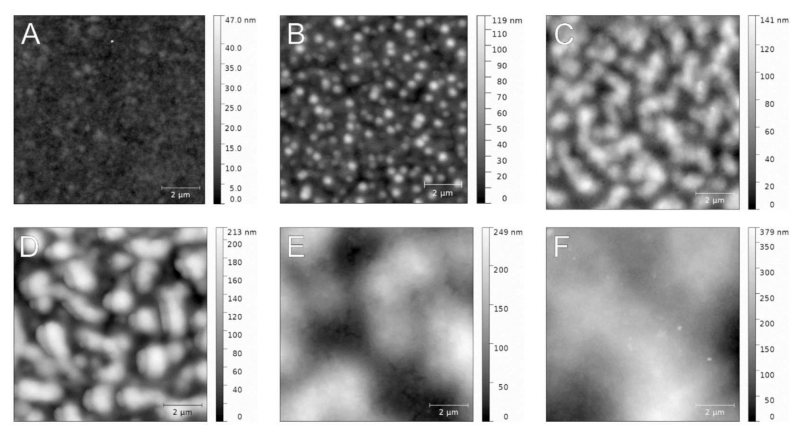

Fig. 2. AFM images selected for the analysis of the samples under study. The images represent samples prepared from solutions with different wt\% loading of fillers: $0,5,10,20,30$, and 40; from (A) to (F), respectively.

TABLE I

Statistical and fractal surface properties of prepared samples, in the same order as in Fig. 2. The parameters are: $f$ [wt\%] - sample filler concentration, $S_{t r}$ - surface anisotropy ratio, $D$ - fractal dimension, $K$ - pseudotopothesy, $\tau_{c}[\mathrm{~nm}]-$ corner frequency, $S_{q}[\mathrm{~nm}]-\mathrm{RMS}$ surface roughness.

\begin{tabular}{c|c|c|c|c|c}
\hline \hline$f$ & $S_{t r}$ & $D$ & $K$ & $\tau_{c}$ & $S_{q}$ \\
\hline 0 & 0.99 & 2.69 & $1.07 \times 10^{-5}$ & 476 & 1.8 \\
5 & 0.97 & 2.23 & $2.21 \times 10^{-3}$ & 350 & 14.8 \\
10 & 0.90 & 2.23 & $3.27 \times 10^{-3}$ & 554 & 25.5 \\
20 & 0.88 & 2.20 & $9.72 \times 10^{-3}$ & 633 & 48.4 \\
30 & 0.98 & 2.35 & $2.30 \times 10^{-3}$ & 1880 & 52.4 \\
40 & 0.60 & 2.46 & $3.00 \times 10^{-3}$ & 2590 & 64.9
\end{tabular}

Table I summarizes the main fractal properties of the images under study. In general, all the samples investigated appear to be monofractal structures, even though the signs of a linear alignment (i.e. secondary structures) can be seen in the $5 \mathrm{wt} \%$ sample. Anisotropy ratio $S_{t r}$ is found to decrease continuously from 0.99 (perfectly isotropic surface) to 0.60 (moderately isotropic surface), with increased filler concentration $f$. This trend is disturbed by the sample with $30 \mathrm{wt} \%$, for which the $S_{t r}$ value goes up to 0.98 . This might be due to the critical point in the concentration of nanofillers reaching saturation of the surface coverage, where linear blocks (contributing to the short-wavelength components of a surface height variations) turn into circular hills with flat sides (contributing to the long-wavelength structures).

A different trend can be seen for the fractal dimension $D$, because this quantity turns out to increase with the $f$ from around 2.20 to 2.46 , except for the first sample (pure polymer), where $D$ equals to 2.69 . The point that a fractal dimension appears also for the sample of pure polymer (see Fig. 2A), and this is the highest of all, can be ascribed to the high sensitivity of AFM and to the probable effect of tiny air bubbles forming in the solution and giving rise to the emerging nanoscale roughness, while we exclude polymer contaminants and dust particles on 
this small size scale. On the other hand, the increasing fractal dimension in the samples of loaded polymer might be explained by the fact that small particles seen in Fig. 2B appear smooth, but when they join to form bigger structures the obtained surface is not as smooth, i.e. the particles leave their fingerprint, influencing the scaling properties. A similar behavior can be seen concerning the changes in the corner frequency $\tau_{c}$, that is the point at which the scaling law disappears. Initially, the turning point occurs at $470 \mathrm{~nm}$ for the pure polymer, falls to $350 \mathrm{~nm}$ for the $5 \mathrm{wt} \%$ sample, but then it gradually increases to $2600 \mathrm{~nm}$ with higher $f$ and increasing size of the growing structures. Increasing amplitude of the height variations is seen in the RMS roughness value $S_{q}$, which continuously increases from 1.8 up to $65 \mathrm{~nm}$ during the sequence of increased $f$.

\section{TABLE II}

Functional surface properties of prepared samples, that involve: $f[\mathrm{wt} \%]$ - sample filler concentration, $S_{k}[\mathrm{~nm}]$ - kernel roughness depth, $S_{p k}[\mathrm{~nm}]$ - reduced peak height, $S_{v k}[\mathrm{~nm}]$ - reduced valley depth, $M_{r 1}\left(M_{r 2}\right)[\%]$ - upper (lower) bearing area.

\begin{tabular}{c|c|c|c|c|c}
\hline \hline$f$ & $S_{k}$ & $S_{p k}$ & $S_{v k}$ & $M_{r 1}$ & $M_{r 2}$ \\
\hline 0 & 4.3 & 12.6 & 3.8 & 13 & 92 \\
5 & 20.1 & 87.6 & 10.1 & 23 & 94 \\
10 & 75.6 & 45.8 & 19.7 & 11 & 98 \\
20 & 129.3 & 68.4 & 15.0 & 16 & 98 \\
30 & 143.9 & 71.4 & 31.6 & 10 & 90 \\
40 & 156.9 & 101.2 & 117.1 & 9 & 88
\end{tabular}

Table II presents the main functional characteristics of the surfaces under study. After all, the kernel roughness depth $S_{k}$ monotonically increases among the samples: from $4.3 \mathrm{~nm}$ (pure polymer) to $157 \mathrm{~nm}$ in the last sample (with the highest $f$ ). As a rule, $S_{k}$ defines that part of the residual surface, which is responsible for its general and long-term tribological behavior. Smaller $S_{k}$ would correspond to higher mechanical resistance and gives higher load-carrying capacity during contacting operations. Reduced peak height $S_{p k}$ is found to be correlated with $S_{k}$, as it raises from $13 \mathrm{~nm}$ to $101 \mathrm{~nm}$ during the increasing concentration sequence. This parameter describes that part of the surface that quickly vanishes upon first contact abrasion; hence it provides information on the running-in properties. Smaller $S_{p k}$ goes hand in hand with shorter running-in times, which is preferable in most engineering applications. The relative amount of the peaks to the overall surface height described by the upper bearing area $M_{r 1}$ changes in a way. Observed samples exhibit $M_{r 1}$ varying between $9 \%$ and $23 \%$, with the lower values observed in the beginning and at the end of the process, while in the middle the $M_{r 1}$ approaches maximum. Increasing values of the reduced valley depth $S_{v k}$ follows the same tendency, going up from $4 \mathrm{~nm}$ to $120 \mathrm{~nm}$ with increasing $f$. $S_{v k}$ specifies the depth of the valleys that should be left after the wearing process, serving as possible lubrication channels. In general, larger
$S_{v k}$ values correspond to better fluid retention properties of the surface. On the other hand, the complement of the $M_{r 2}$ to $100 \%$ actually depicts the percentage of the valleys on the surface, and its value varying between $2 \%$ and $12 \%$ is generally lower than $M_{r 1}$.

\section{Conclusion}

This study of the AFM images of samples of thin films made of nanocomposites with the advanced mathematical tools selected here, namely advanced statistical parameters of the height distributions and fractal geometry, confirms that such tools can provide insight into surface morphology characteristics that may turn out to be functional for the analyzed materials. In particular, here we focused our discussion on the possible application of our films as coatings of different bulk materials. In fact, these materials could be used for paints or functional film aiming to take advantage of the photocatalytic properties of the fillers for e.g. chemical or bacterial pollution of air or liquids in contact with this surface. In this application, the scratch resistance of the film is of major importance, which can be connected to the detailed surface morphology arising from the coating technique. The results of our analysis can represent a starting point and a guide for subsequent wear tests on similar samples. Overall, it is confirmed that the films surface morphology depends on the material composition, and that the statistical surface roughness parameters and fractal analysis may provide useful insight into the nature of the composite thin films. These results can be used to develop mathematical theoretical models which help to study structure, simulation of dynamics at interfaces, and thermodynamics processes at nanometer level.

\section{References}

[1] R. Bogue, Assem. Autom. 31, 106 (2011).

[2] S. Thorat, A. Diaspro, M. Salerno, Adv. Mater. Lett. 4, 15 (2013).

[3] I.-Y. Jeon, J.-B. Baek, Materials (Basel) 3, 3654 (2010).

[4] E. Habib, R. Wang, Y. Wang, M. Zhu, X.X. Zhu, ACS Biomater. Sci. Eng. 2, 1 (2016).

[5] S.B. Thorat, A. Diaspro, M. Salerno, J. Dent. 42, 279 (2014).

[6] S.R. Bakshi, D. Lahiri, A. Agarwal, Int. Mater. Rev. 55, 41 (2010).

[7] J.E. Mates, I.S. Bayer, M. Salerno, P.J. Carroll, Z. Jiang, L. Liu, C.M. Megaridis, Carbon (New York) 87, 163 (2015).

[8] T. Kuilla, S. Bhadra, D. Yao, N.H. Kim, S. Bose, J.H. Lee, Prog. Polym. Sci. 35 , 1350 (2010).

[9] X. Chen, S.S. Mao, Chem. Rev. 107, 2891 (2007).

[10] A.P. Reverberi, N.T. Kuznetsov, V.P. Meshalkin, M. Salerno, B. Fabiano, Teor. Osn. Khim. Tekhnol. 50, 63 (2016).

[11] A. Shayganpour, A. Rebaudi, P. Cortella, A. Diaspro, M. Salerno, Beilstein J. Nanotechnol. 6, 2183 (2015). 
[12] R. Carzino, F. Pignatelli, D. Farina, B. Torre, M. Scotto, L. Marini, G. Bertoni, G. Caputo, P.D. Cozzoli, A. Diaspro, A. Athanassiou, Nanotechnology 25, 125702 (2014).

[13] F. Pignatelli, R. Carzino, M. Salerno, M. Scotto, C. Canale, M. Distaso, F. Rizzi, G. Caputo, P.D. Cozzoli, R. Cingolani, A. Athanassiou, Thin Solid Films 518, 4425 (2010).

[14] Ş. Tălu, N. Patra, M. Salerno, Prog. Org. Coat. 89, 50 (2015)

[15] S. Tălu, S. Stach, S.F. Alb, M. Salerno, Chaos Solit. Fract. 71, 7 (2015).

[16] Ş. Ţălu, Micro and Nanoscale Characterization of Three Dimensional Surfaces. Basics and Applications, Napoca Star Publ. House, Cluj-Napoca 2015.

[17] R. Shikhgasan, S. Dinara, S. Sebastian, R. Guseyn, Superlatt. Microstruct. 86, 395 (2015).

[18] S. Stach, D. Dallaeva, S. Talu, P. Kaspar, P. Tomanek, S. Giovanzana, L. Grmela, Mater. Sci. (Poland) 33, 175 (2015)
[19] G. Caputo, B. Cortese, C. Nobile, M. Salerno, R. Cingolani, G. Gigli, P. Cozzoli, A. Athanassiou, Adv. Funct. Mater. 19, 1149 (2009).

[20] S. Kulesza, M. Bramowicz, Appl. Surf. Sci. 293, 196 (2014).

[21] S. Ţălu, M. Bramowicz, S. Kulesza, A. Shafiekhani, A. Ghaderi, F. Mashayekhi, S. Solaymani, Ind. Eng. Chem. Res. 54, 8212 (2015).

[22] P. Czaja, W. Maziarz, J. Przewoźnik, A. Zywczak, P. Ozga, M. Bramowicz, S. Kulesza, J. Dutkiewicz, Intermetallics 55, 1 (2014).

[23] W.P. Dong, P.J. Sullivan, K.J. Stout, Wear 178, 45 (1994).

[24] R.S. Sayles, T.R. Thomas, Wear 42, 263 (1977).

[25] A. Thomas, T.R. Thomas, J. Wave Mater. Interact. 3, 341 (1988) 